\title{
REMARKS ON THE EXTENSION OF THE RICCI FLOW
}

\author{
FEI HE
}

\begin{abstract}
We present two new conditions to extend the Ricci flow on a compact manifold over a finite time, which are improvements of some known extension theorems.
\end{abstract}

\section{INTRODUCTION}

We say that $g(t)$ is a Ricci flow solution if it satisfies the following equation defined by Richard Hamilton [5] :

$$
\frac{\partial}{\partial t} g(t)=-2 \operatorname{Ric}(t) .
$$

In the following $R m(t), R i c(t)$ and $R(t)$ denote the Riemann, Ricci and scalar curvature tensors of $g(t)$ respectively, and $|R m(t)|,|R i c(t)|$ denote the corresponding norms.

The Ricci flow equation (1.1) has been studied extensively. Short time existence of solutions was first established by R. Hamilton in [5]. Convergence of solutions to Einstein metrics is proved possible for initial metrics with special curvature conditions. In general, the Ricci flow solution will develop singularities in finite or infinite time. Therefore an important topic in the theory is the behavior of curvature tensors at a singular time.

R. Hamilton showed in [6] that if $T<\infty$ is a finite singular time, we have

$$
\limsup _{t \rightarrow T} \sup _{M}|R m(t)|=+\infty .
$$

In other words, if the sectional curvature is uniformly bounded on a finite time interval, then the flow can be extended ([5]). The proof is by establishing BernsteinBando type smoothing estimates using the maximum principle. Hamilton's theorem has been improved by Natasa Sesum who showed that if

$$
\sup _{M \times[0, T)}|\operatorname{Ric}(t)|<\infty,
$$

then the Ricci flow can be extended past time $T<\infty$ ([16]). These results are known as extension theorems for the Ricci flow.

A natural question is: what is the weakest curvature condition to extend the Ricci flow? There is a conjecture that in a finite time singularity of the Ricci flow, the supremum of the scalar curvature will blow up. This conjecture is confirmed for Type I singularities (44, 19]) and for the Kähler Ricci flow ([25]). But the general case is still open. One of the best known results in this direction is the following Theorem [1.1. which is implied by the proof method of Natasa Sesum in [16, and is also proved by Bing Wang in [22]:

Date: November 5, 2018.

This research was partially supported by NSF grant DMS-0801988. 
Theorem 1.1. Suppose $(M, g(t)), 0 \leq t<T<\infty$, is a Ricci flow solution on a closed manifold. If

$$
\int_{0}^{T} \sup _{M}|\operatorname{Ric}(t)| d t<+\infty .
$$

Then the flow can be extended past time $T$.

Remark 1. Bing Wang also proved in 22$]$ that there is a gap for $\lim \sup _{t \rightarrow T}|T-t| \sup _{M}|\operatorname{Ric}(t)|$ where $T$ is the singular time.

Theorem 1.1 tells that $\sup _{M}|\operatorname{Ric}(t)|$ not only blows up at a finite singular time, but also has to grow fast enough so that its integral on the maximal existence time interval is infinite. This clearly recovers previous results mentioned above.

Our first theorem is a further improvement in this direction. The new idea is to explore the optimal Sobolev constant and apply a related rigidity theorem.

Theorem 1.2. Suppose $(M, g(t)), 0 \leq t<T$, is a Ricci flow solution on a closed manifold, $T<\infty$. If the function $F(x):=\int_{0}^{T}|\operatorname{Ric}(x, t)| d t$ is continuous on $M$, then the flow can be extended past time $T$.

Remark 2. Note that if $\int_{0}^{T} \sup _{M}|\operatorname{Ric}(t)| d t<\infty$, the dominated convergence theorem implies the continuity of $\int_{0}^{T}|\operatorname{Ric}(x, t)| d t$, and we recover Theorem 1.1

The proof of Theorem 1.2 uses a blow-up argument. Recall that by Hamilton's compactness theorem ([7]) and Perelman's no-local-collapsing theorem ([15), we can choose a sequence of times and points $\left(x_{i}, t_{i}\right) \in M \times[0, T), i=1,2, \ldots$, where $t_{i} \rightarrow T$, such that the sequence of dilated pointed solutions $\left(M, g_{i}(t), x_{i}\right)$ with $g_{i}(t)$ defined by

$$
g_{i}(t):=\left|R m\left(x_{i}, t_{i}\right)\right| g\left(t_{i}+\frac{t}{\left|R m\left(x_{i}, t_{i}\right)\right|}\right)
$$

converges in the pointed Cheeger-Gromov sense to a complete limit solution $\left(M_{\infty}\right.$, $\left.g_{\infty}(t), x_{\infty}\right), t \in(-\infty, \omega)$, where $\omega$ is a positive number or $\infty$. It's important that this limit solution is non-flat when $T$ is a finite singular time, in particular $\left|R m\left(x_{\infty}, 0\right)\right|=1$. This compactness result is very useful in studying the behavior of the Ricci flow at a singular time. For example, recall that ([16]) Natasa Sesum studied the volume growth of geodesic balls in $\left(M_{\infty}, g_{\infty}(0)\right)$, and used the rigidity part of the volume comparison theorem to conclude that, if $|\operatorname{Ric}(t)|$ is uniformly bounded for $t \in[0, T)$, then $\left(M_{\infty}, g_{\infty}(0)\right)$ is isometric to the Euclidean space, hence contradicting with the non-flatness.

Under the assumption of Theorem 1.2, we can establish an optimal Euclidean Sobolev inequality on $\left(M_{\infty}, g_{\infty}(0)\right)$, then apply the following rigidity theorem of M. Ledoux to show that $\left(M_{\infty}, g_{\infty}(0)\right)$ is isometric to the Euclidean space.

Theorem 1.3 (M. Ledoux, 13). Let $(M, g)$ be a smooth, complete $n$-dimensional Riemannian manifold with nonnegative Ricci curvature. Suppose that for some $q \in[0, n)$, the Sobolev inequality

$$
\left(\int_{M}|u|^{p} d \mu\right)^{q / p} \leq K(n, q)^{q} \int_{M}|\nabla u|^{q} d \mu
$$

is valid for $\forall u \in C_{0}^{\infty}(M)$, where $1 / p=1 / q-1 / n, K(n, q)$ is the optimal Sobolev constant for the Euclidean space. Then $(M, g)$ is isometric to $\left(\mathbb{R}^{n}, g_{\text {flat }}\right)$.

Remark 3. The value $K(n, q)$ is computed in [20]. 
Optimal constants in Sobolev inequalities have been studied by many authors, and one can refer to [8] for a comprehensive exposition. In the proof we need a theorem of T. Aubin [1]:

Theorem 1.4 (T. Aubin). Let $(M, g)$ be a smooth, compact Riemannian n-manifold. For any $\epsilon>0$ and any $q \in[1, n)$ real, there exists $B \in \mathbb{R}$ such that for any $u \in H_{1}^{q}(M)$,

$$
\left(\int_{M}|u|^{p} d \mu\right)^{q / p} \leq\left(K(n, q)^{q}+\epsilon\right) \int_{M}|\nabla u|^{q} d \mu+B \int_{M} u^{q} d \mu
$$

where $1 / p=1 / q-1 / n$ and $K(n, q)$ is the optimal Sobolev constant for the Euclidean space.

Remark 4. T. Aubin's theorem has been improved by E. Hebey and M.Vaugon (9], [10], ) who showed that the $\epsilon$ in Theorem 1.4 can be removed, in both compact and complete settings.

Remark 5. T. Aubin's theorem implies that for any $\epsilon>0$, we have a family of Sobolev inequalities in the form

$$
\left(\int_{M}|u|^{2 n /(n-2)} d \mu(t)\right)^{(n-2) / n} \leq\left(K(n, 2)^{2}+\epsilon\right) \int_{M}|\nabla u|_{g(t)}^{2} d \mu(t)+B(t) \int_{M} u^{2} d \mu(t),
$$

along the flow. The proof of Theorem 1.2 implies that $B(t)$ must blow up at a finite singular time. It will be very interesting to get an upper bound estimate of $B(t)$ explicitly in terms of curvature, however such an estimate is not yet available to our knowledge.

Space-time integral bounds on the curvature have also been considered by many authors. In 21] B. Wang proved that if

$$
\int_{0}^{T} \int_{M}|R m|^{p} d \mu d t<\infty, \quad p \geq \frac{n}{2}+1,
$$

then the Ricci flow can be extended past time $T$. Similar results are also proved in 23] by R. Ye and in [14] by L. Ma and L. Cheng. Note that the power $\frac{n}{2}+1$ is critical, which makes the integral scaling invariant. If $p<\frac{n}{2}+1$, the integral in (1.2) can be bounded even when $T$ is a singular time, as demonstrated by the shrinking sphere (See Example 2.1 in [21]).

For the mean curvature flow, the same extension problem has also been studied. The supremum and certain scaling invariant space-time integrals of the norm of the second fundamental form are known to blow up at a finite singular time (11], [17, [18], 24]). Moreover, the surprising fact that a subcritical integral quantity has to blow up was proved by N. Le in [12]:

Theorem 1.5 (N.Le). Let $A(t)$ be the second fundamental form of a $n$-dimensional compact hyper-surface without boundary $M_{t}$ in $\mathbb{R}^{n+1}$ evolving by the mean curvature flow. If

$$
\int_{0}^{T} \int_{M_{t}} \frac{|A|^{n+2}}{\log (1+|A|)} d \mu d t<\infty
$$

then the flow can be extended past time $T$. 
Note that $n+2$ is the critical power in the mean curvature flow case, and that the integral quantity in N. Le's theorem is sub-scaling invariant.

One of the key elements in the proof is the Michael-Simon inequality, which one uses to establish a Sobolev inequality, then applying Nash-Moser iteration to prove the following mean-value type inequality:

$$
\sup _{M_{t}}|A(t)| \leq C\left(M_{0}\right)\left(1+\int_{0}^{T} \int_{M_{t}}|A|^{n+3}\right) .
$$

Theorem 1.5 then follows an elementary calculus method.

Our second result is a Ricci flow version of N. Le's theorem.

Theorem 1.6. Let $(M, g(t)), t \in[0, T)$ be a Ricci flow solution. If

$$
\int_{0}^{T} \int_{M} \frac{|R m|^{n / 2+1}}{\log (1+|R m|)} d \mu d t<\infty
$$

Then the flow can be extended past time $T$.

In the Ricci flow case, we can use a blow-up argument and apply the 'doublingtime estimate' (Corollary 7.5 in [2]) to establish an inequality similar to (1.3), then use the same calculus method to prove Theorem 1.6 .

\section{Proof of Theorem 1.2}

Proof of Theorem 1.2. We claim that under the assumption of the theorem, the sectional curvature $|R m|$ is bounded, hence the flow can be extended by Hamilton's result (Theorem 14.1 in [5]).

If the claim is not true, we can choose a sequence of times and points $\left(x_{i}, t_{i}\right) \in$ $M \times[0, T), i=1,2, \ldots$, such that $Q_{i}:=\left|R m\left(x_{i}, t_{i}\right)\right| \rightarrow \infty$ and $t_{i} \rightarrow T$ as $i \rightarrow \infty$, and the sequence of dilated pointed solutions $\left(M, g_{i}(t), x_{i}\right)$ with $g_{i}(t)$ defined by

$$
g_{i}(t):=Q_{i} g\left(t_{i}+\frac{t}{Q_{i}}\right)
$$

converges in the pointed Cheeger-Gromov sense to a non-flat limit solution $\left(M_{\infty}, g_{\infty}\right.$, $x_{\infty}$ ) (See Chapter 8 of [2]). In the following we use $\phi_{i}, i=1,2, \ldots$ to denote the diffeomorphisms in the pointed Cheeger-Gromov limit ( See Chapter 3 of [3] for a detailed definition). Also, we use $R^{+}$and $R^{-}$to denote the positive and negative parts of the scalar curvature, and $\lambda$ is the negative part of the smallest eigenvalue of the Ricci curvature.

We first prove that $\left(M_{\infty}, g_{\infty}\right)$ has nonnegative Ricci curvature (We actually prove it is Ricci-flat). By the continuity assumption on $F(x):=\int_{0}^{T}|\operatorname{Ric}(x, t)| d t$ and the compactness of $M$, we can use elementary arguments to prove that

$$
\lim _{s \rightarrow T} \int_{s}^{T}|\operatorname{Ric}(x, t)| d t=0 \quad \text { uniformly for } \quad \forall x \in M .
$$

Then we compute

$$
\begin{aligned}
\int_{-1}^{0}\left|R i c_{g_{\infty}(t)}\right|(x) d t & =\lim _{i \rightarrow \infty} \int_{-1}^{0}\left|R i c_{\phi_{i}^{*} g_{i}(t)}\right|(x) d t \\
& =\lim _{i \rightarrow \infty} \int_{-1}^{0}\left|R i c_{g_{i}(t)}\right|\left(\phi_{i}(x)\right) d t
\end{aligned}
$$




$$
\begin{aligned}
& =\lim _{i \rightarrow \infty} \int_{t_{i}-1 / Q_{i}}^{t_{i}}\left|\operatorname{Ric}_{g(t)}\right|\left(\phi_{i}(x)\right) d t \\
& \leq \lim _{i \rightarrow \infty} \int_{t_{i}-1 / Q_{i}}^{t_{i}}\left|R i c_{g(t)}\right|\left(\phi_{i}(x)\right) d t \\
& \leq \lim _{i \rightarrow \infty} \int_{t_{i}-1 / Q_{i}}^{T}\left|\operatorname{Ric}_{g(t)}\right|\left(\phi_{i}(x)\right) d t \\
& =0 .
\end{aligned}
$$

Which implies $\left|\operatorname{Ric}_{g_{\infty}(t)}\right|(x)=0, \forall x \in M_{\infty}, \forall t \in[-1,0]$.

Next we establish a Sobolev inequality on $M_{\infty}$. Observe that

$$
\frac{d}{d t}|\nabla u|_{g}^{2}(t)(x)=2 \operatorname{Ric}(t)(\nabla u, \nabla u)
$$

and

$$
\frac{d}{d t} d \mu_{g(t)}(x)=-R(x, t) d \mu_{g(t)}(x)
$$

These imply that

$$
|\nabla u|^{2}\left(x, t_{0}\right) \leq|\nabla u|^{2}\left(x, t_{1}\right) e^{2 \int_{t_{0}}^{t_{1}} \lambda(x, t) d t}
$$

and

$$
e^{-\int_{t_{0}}^{t_{1}} R^{+}(x, t) d t} d \mu\left(x, t_{0}\right) \leq d \mu\left(x, t_{1}\right) \leq e^{\int_{t_{0}}^{t_{1}} R^{-}(x, t) d t} d \mu\left(x, t_{0}\right) .
$$

Now we need the continuity of $F(x):=\int_{0}^{T}|\operatorname{Ric}(x, t)| d t$ and the compactness of $M$ again. For any $\epsilon>0$, by elementary analysis we can find $t_{0}(\epsilon)$ such that $\forall t_{2}>t_{1} \geq t_{0}$, we have

$$
\begin{array}{r}
0 \leq \int_{t_{1}}^{t_{2}} R^{-}(x, t) d t \leq n \int_{t_{1}}^{t_{2}}|\operatorname{Ric}(x, t)| d t<\epsilon ; \\
0 \leq \int_{t_{1}}^{t_{2}} R^{+}(x, t) d t \leq n \int_{t_{1}}^{t_{2}}|\operatorname{Ric}(x, t)| d t<\epsilon ; \\
\quad 0 \leq \int_{t_{1}}^{t_{2}} \lambda(x, t) d t \leq \int_{t_{1}}^{t_{2}}|\operatorname{Ric}(x, t)| d t<\epsilon ;
\end{array}
$$

for all $x \in M$.

Theorem 1.4 implies that we have a Sobolev inequality at the time $t_{0}$ :

$$
\left(\int_{M}|u|^{2 n /(n-2)} d \mu\left(t_{0}\right)\right)^{(n-2) / n} \leq\left(K(n, 2)^{2}+\epsilon\right) \int_{M}|\nabla u|_{g\left(t_{0}\right)}^{2} d \mu\left(t_{0}\right)+B\left(t_{0}\right) \int_{M} u^{2} d \mu\left(t_{0}\right),
$$

for any $u \in H_{1}^{2}(M)$. Then the above observation implies that for any $t_{1} \in\left[t_{0}, T\right)$, $\left(M, g\left(t_{1}\right)\right)$ has a Sobolev inequality:

$$
\begin{aligned}
\left(\int_{M}|u|^{2 n /(n-2)} d \mu\left(t_{1}\right)\right)^{(n-2) / n} \leq & \left(K(n, 2)^{2}+\epsilon\right) e^{(3-2 / n) \epsilon} \int_{M}|\nabla u|_{g\left(t_{1}\right)}^{2} d \mu\left(t_{1}\right) \\
& +B\left(t_{0}\right) e^{(2-2 / n) \epsilon} \int_{M} u^{2} d \mu\left(t_{1}\right)
\end{aligned}
$$

for any $u \in H_{1}^{2}(M)$.

Now we pick any $u \in C_{0}^{\infty}\left(M_{\infty}\right)$, and suppose $u$ is supported on a compact domain $V$. The idea is to push $u$ forward by $\phi_{i}$ to a function on $\left(M, g\left(t_{i}\right)\right)$ for each 
$i$ s.t. $t_{i}>t_{0}$, apply the Sobolev inequality, then pull back to $\left(M_{\infty}, g_{\infty}(0)\right)$ and take the limit in $i$. We compute:

$$
\begin{aligned}
& \left(\int_{M_{\infty}}|u|^{2 n /(n-2)} d \mu_{g_{\infty}(0)}\right)^{(n-2) / n} \\
= & \lim _{i \rightarrow \infty}\left(\int_{V}|u|^{2 n /(n-2)} d \mu_{\phi_{i}^{*} g_{i}(0)}\right)^{(n-2) / n} \\
= & \lim _{i \rightarrow \infty}\left(Q_{i}^{n / 2} \int_{\phi_{i}(V)}\left|u \circ \phi_{i}^{-1}\right|^{2 n /(n-2)} d \mu_{g\left(t_{i}\right)}\right)^{(n-2) / n} \\
\leq & \lim _{i \rightarrow \infty} Q_{i}^{(n-2) / n}\left[\left(K(n, 2)^{2}+\epsilon\right) e^{(3-2 / n) \epsilon} \int_{\phi_{i}(V)}\left|\nabla\left(u \circ \phi_{i}^{-1}\right)\right|_{g\left(t_{i}\right)}^{2} d \mu_{g\left(t_{i}\right)}\right. \\
& \left.+B\left(t_{0}\right) e^{(2-2 / n) \epsilon} \int_{\phi_{i}(V)} u^{2} d \mu_{g\left(t_{i}\right)}\right] \\
= & \lim _{i \rightarrow \infty}\left[\left(K(n, 2)^{2}+\epsilon\right) e^{(3-2 / n) \epsilon} \int_{\phi_{i}(V)}\left|\nabla\left(u \circ \phi_{i}^{-1}\right)\right|_{g_{i}(0)}^{2} d \mu_{g_{i}(0)}\right. \\
& \left.+\frac{B\left(t_{0}\right) e^{(2-2 / n) \epsilon}}{Q_{i}} \int_{\phi_{i}(V)} u^{2} d \mu_{g_{i}(0)}\right] \\
= & \left(K(n, 2)^{2}+\epsilon\right) e^{(3-2 / n) \epsilon} \int_{M_{\infty}}|\nabla u|_{g_{\infty}(0)}^{2} d \mu_{g_{\infty}(0)} .
\end{aligned}
$$

Since $\epsilon$ is arbitrary, we can let it go to zero. Then we establish the optimal Euclidean Sobolev inequality

$$
\left(\int_{M_{\infty}}|u|^{2 n /(n-2)} d \mu_{g_{\infty}(0)}\right)^{(n-2) / n} \leq K(n, 2)^{2} \int_{M_{\infty}}|\nabla u|_{g_{\infty}(0)}^{2} d \mu_{g_{\infty}(0)}
$$

on $\left(M_{\infty}, g_{\infty}(0)\right)$.

By Theorem 1.3. $\left(M_{\infty}, g_{\infty}(0)\right)$ is isometric to the Euclidean space, contradicting the non-flatness of $g_{\infty}(0)$.

\section{Proof of Theorem 1.6}

To prove Theorem 1.6. we first establish a similar inequality to (1.3) by a compactness argument. We need the following 'doubling-time estimate', which is Corollary 7.5 in $[2]$ :

Lemma 3.1 (Doubling-time estimate). There exists $c(n)$ depending only on $n$, such that if $(M, g(t), t \in[0, T))$ is a Ricci flow solution on a compact manifold of dimension $n$, then

$$
\sup _{M}|R m(t)| \leq 2 \sup _{M}|R m(0)| \quad \text { for all times } t \in\left[0, \frac{c(n)}{\sup _{M}|R m(0)|}\right) .
$$

Lemma 3.2. Let $\mathcal{M}=\{g(t) \mid t \in[0,1], \quad g(t)$ has non-collapsing constant $\left.\kappa, \sup _{M}|R m(0)| \leq C_{0}\right\}$ be a set of nonsingular Ricci flow solutions on a closed $n$-dimensional manifold $M$. There exists a constant $C\left(n, \kappa, C_{0}\right)$ such that for any 
$g(t) \in \mathcal{M}$

$$
\sup _{M \times[0,1]}|R m| \leq C \int_{0}^{1} \int_{M}|R m|^{n / 2+2} d \mu d t+2 C_{0}
$$

Proof. If not, we can find a sequence $g_{i}(t), i=1,2, \ldots$ in $\mathcal{M}$, such that

$$
\sup _{M \times[0,1]}\left|R m_{i}\right| \geq P_{i} \int_{0}^{1} \int_{M}\left|R m_{i}\right|^{n / 2+2} d \mu_{i} d t+2 C_{0},
$$

where $P_{i} \rightarrow+\infty$ as $i \rightarrow \infty$. Let $Q_{i}=\sup _{M \times[0,1]}\left|R m_{i}\right|$ for each $i$, then we can find $\left(x_{i}, t_{i}\right)$ such that $Q_{i}=\left|R m_{i}\left(x_{i}, t_{i}\right)\right|$. Note that $Q_{i}>2 C_{0}$, Lemma 3.1 implies that $t_{i} \geq c(n) / C_{0}$, hence $Q_{i} t_{i} \geq 2 c(n)$. Dilate this sequence

$$
\tilde{g}_{i}(t)=Q_{i} g_{i}\left(t_{i}+t / Q_{i}\right),-t_{i} Q_{i} \leq t \leq\left(1-t_{i}\right) Q_{i}, i=1,2, \ldots
$$

The dilated solutions $\left(M, \tilde{g}_{i}(t), x_{i}\right)$ has a common existence interval $\left[-2 C_{0}, 0\right]$, a uniform bound on the curvature and an injectivity radius lower bound by the assumption on the non-collapsing constant $\kappa$. Hence they converge in the pointed CheegerGromov sense to a limit solution $\left(M_{\infty}, \tilde{g}(t), x_{\infty}\right), t \in\left[-2 C_{0}, 0\right]$, with $|\widetilde{R m}|\left(x_{\infty}, 0\right)=$ 1. But we can compute on any $x_{\infty} \in \Omega \subset M_{\infty}$

$$
\begin{aligned}
& \int_{-2 C_{0}}^{0} \int_{\Omega}|\widetilde{R m}|^{n / 2+2} d \tilde{\mu} d t \\
= & \lim _{i \rightarrow \infty} 1 / Q_{i} \int_{t_{i}-2 c(n) / Q_{i}}^{t_{i}} \int_{\phi_{i}(\Omega)}\left|R m_{i}\right|^{n / 2+2} d \mu_{i} d t \\
\leq & \lim _{i \rightarrow \infty}\left(\frac{1}{Q_{i}} \frac{Q_{i}-2 C_{0}}{P_{i}}\right) \\
= & 0 .
\end{aligned}
$$

Which implies that $|\widetilde{R m}|\left(x_{\infty}, 0\right)=0$, contradiction!

Lemma 3.3 (Mean Value Inequality). For a Ricci flow solution $(M, g(t)), t \in$ $[0, T), T<\infty$, there exists constants $C_{0}\left(n, \kappa, \sup _{M}|R m(0)|\right)$ and $C_{1}=T \max \left\{2 \sup _{M}|R m(0)|, 2 \sup _{M}|R m(0)|^{2} / c(n)\right\}$, where $c(n)$ is the constant in the 'doubling-time estimate', such that for any $t \in[0, T)$

$$
\sup _{M \times[0, t]}|R m| \leq C_{0} \int_{0}^{t} \int_{M}|R m(x, s)|^{n / 2+2} d \mu d s+C_{1} .
$$

Proof. We only need to prove the lemma for non-trivial solutions. Without loss of generality let $T=1$.

For $t \in\left[0, c(n) / \sup _{M}|R m(0)|\right)$ it's clearly true by Lemma 3.1.

For any $t \in\left[c(n) / \sup _{M}|R m(0)|, 1\right)$, define

$$
\tilde{g}(s)=\frac{1}{t} g(t s), \quad s \in[0,1] .
$$

Then

$$
|\widetilde{R m}(0)| \leq t|R m(0)| \leq|R m(0)| .
$$

Note that the non-collapsing constant $\kappa$ is scaling invariant. Lemma 3.2 implies

$$
\sup _{M \times[0, t]}|\widetilde{R m}| \leq C_{0} \int_{0}^{t} \int_{M}|\widetilde{R m}(x, s)|^{n / 2+2} d \tilde{\mu} d s+2 \sup _{M}|R m(0)| .
$$


Then we scale it back to the original metric $g(t)$. Since the scaling factor $t$ is now bounded below by $c(n) / \sup _{M}|R m(0)|$, we get

$$
\sup _{M \times[0, t]}|R m| \leq C_{0} \int_{0}^{t} \int_{M}|R m(x, s)|^{n / 2+2} d \mu d s+2 \sup _{M}|R m(0)|^{2} / c(n) .
$$

Now we can use the same method as in [12] to prove Theorem 1.6.

Proof of Theorem 1.6. Let

$$
\begin{gathered}
f(t)=\sup _{M}|R m(t)|, \\
G(t)=\int_{M} \frac{|R m|^{n / 2+1}}{\log (1+|R m|)} d \mu(t),
\end{gathered}
$$

and

$$
\psi(s)=s \log (1+s) .
$$

Then $\psi$ is an increasing function when $s \geq 0$. By Lemma 3.3 for any $t \in[0, T)$

$$
\begin{aligned}
f(t) & \leq C \int_{0}^{t} \int_{M} \psi(|R m|) \frac{|R m|^{n / 2+1}}{\log (1+|R m|)} d \mu d s+C_{1} \\
& \leq C \int_{0}^{t} \psi(f(s)) G(s) d s+C_{1} \\
& =: h(t) .
\end{aligned}
$$

$h^{\prime}(t)=C \psi(f(t)) G(t) \leq C \psi(h(t)) G(t)$ since $\psi$ is nondecreasing. Then we have

$$
\begin{aligned}
\int_{h(0)}^{h(t)} \frac{1}{\psi(s)} d s & =\int_{0}^{t} C G(t) d t \\
& \leq C \int_{0}^{t} \int_{M} \frac{|R m|^{n / 2+1}}{\log (1+|R m|)} d \mu d t \\
& <\infty .
\end{aligned}
$$

Since $\int_{1}^{\infty} \frac{1}{\psi(s)} d s=\infty$, we deduce that $\sup _{[0, T)} h(t)<\infty$, hence $\sup _{[0, T)} f(t)<$ $\infty$. Therefore the flow can be extended by Theorem 14.1 in [5].

Acknowledgements: The author would like to thank his advisor Peter Li for his advising, encouragement and generous support. Also thank Jeffrey Streets for many useful suggestions and all his help in preparing this paper, and thank Guoyi $\mathrm{Xu}$ for helpful discussions.

\section{REFERENCES}

1. T. Aubin, Problèmes isopérimétriques et espaces de Sobolev; : J. Differential Geom. Volume 11, Number 4 (1976), 573-598.

2. Bennet Chow and Dan Knopf, The Ricci Flow: An Introduction; Mathematical Surveys and Monographs, Vol. 110. American Mathematical Society, Providence, RI, 2004.

3. Bennet Chow, et al. The Ricci Flow: Techniques and Applications, Part I: Geometric Aspects; Mathematical Surveys and Monographs, Vol. 135. American Mathematical Society, Providence, RI, 2007.

4. Joerg Enders, Reto Müller, Peter M. Topping, On Type I Singularities in Ricci flow; arXiv:1005.1624 1 [math.DG].

5. R.S.Hamilton,Three-manifolds with positive Ricci curvature; J. Differential Geom. Volume 17, Number 2 (1982), 255-306. 
6. --, The formation of singularities in the Ricci flow, Surveys in differential geometry, Vol. II, pp. 7-136, International Press, Cambridge, MA, 1995.

7. --, A compactness property for solutions of the Ricci flow, Amer. J. Math. 117 (1995) 545572

8. E. Hebey, Nonlinear Analysis on manifolds: Sobolev Spaces and Inequalities, Courant Lecture Notes in Mathematics, vol. 5, New York University Courant Institute of Mathematical Sciences, New York (1999).

9. E. Hebey and M. Vaugon, Meilleures constantes dans le theoreme d'inclusion de Sobolev, CR. Acad. Sci. Paris Ser. I Math. 318 (1994), 675-677.

10. --, The best constant problem in the Sobolev embedding. Duke math. J. 79(1), 235-279 (1995).

11. Huisken, G., Flow by mean curvature of convex surfaces into spheres; J. Diferential Geom. 20 (1984), no. 1, 237-266.

12. Nam Q.Le, Blow Up of Subcritical Quantities at The First Singular Time of The Mean Curvature Flow; arXiv: 1002.4669v2.

13. M. Ledoux, On manifolds with non-negative Ricci curvature and Sobolev inequalities.Comm. Anal. Geom. 7 (1999), 347-353.

14. Li Ma and Liang Cheng. On the conditions to control curvature tensors of Ricci flow. Analysis and Geometry 37(2010), no.4, 403-411.

15. G. Perelman, The entropy formula for the Ricci flow and its geometric applications, arXiv:math.DG/0211159

16. Natasa Sesum, Curvature tensor under the Ricci flow; Amer. J. Math. 127 (2005), no.6, 13151324

17. Nam Q.Le, Natasa Sesum, On the extension of the mean curvature flow, Math. Z. DOI:10.1007/s00209-009-0637-1.

18. --, The mean curvature at the first singular time of the mean curvature flow, arXiv:1001.3682 1 [math.DG].

19. --, Remarks on curvature behavior at the first singular time of the Ricci flow; arXiv:1005.1220 2 [math.DG]

20. G. Talenti, Best constant in Sobolev inequality, Ann. Mat. PuraAppl. 110 (1976), 353-372.

21. Bing Wang, On the conditions to extend Ricci flow; arXiv:0704.3018v2.

22. _-, On the conditions to extend Ricci flow(II); arXiv:1107.5107 1.

23. Rugang Ye, Curvature estimates for the Ricci flow, II, Cal. Var. Partial Differential Equations, 31(4):439-455, 2008.

24. H. W. Xu, F. Ye, E. T. Zhao, Extend Mean Curvature Flow with Finite Integral Curvature, arXiv:0905.1167v1.

25. Zhou Zhang, Scalar curvature behavior for finite-time singularity of Kähler-Ricci flow, Michigan Math. J. 59 (2010), no. 2, 419C433.

Department of Mathematics, University of California, Irvine, CA 92697

E-mail address: hef@uci.edu 